\title{
Erratum to: Applied Intelligence, Volume 31 (1)
}

The Publisher

Published online: 6 November 2009

(C) Springer Science+Business Media, LLC 2009

Erratum to: Appl Intell (2009) 31:1-14

DOI 10.1007/s10489-007-0105-8

Appl Intell (2009) 31:15-30

DOI 10.1007/s10489-007-0107-6

Appl Intell (2009) 31:81-88

DOI 10.1007/s10489-008-0114-2

The following three papers were scheduled to be part of the Special Issue on Learning and Modeling in Intelligent Applications but inadvertently published in an earlier issue, Volume 31, Number 1.

Springer regrets the error.

Automated debugging of recommender user interface descriptions

DOI 10.1007/s10489-007-0105-8

Alexander Felfernig, Gerhard Friedrich, Klaus Isak, Kostyantyn Shchekotykhin, Erich Teppan and Dietmar Jannach
Improving fuzzy logic controllers obtained by experts: a case study in HVAC systems

DOI 10.1007/s10489-007-0107-6

Rafael Alcalá, Jesús Alcalá-Fdez, María José Gacto and Francisco Herrera

Locality kernels for sequential data and their applications to parse ranking

DOI 10.1007/s10489-008-0114-2

Evgeni Tsivtsivadze, Tapio Pahikkala, Jorma Boberg and Tapio Salakoski

The online versions of the original articles can be found under doi:

10.1007/s10489-007-0105-8, 10.1007/s10489-008-0114-2, 10.1007/s10489-007-0107-6.

The Publisher

http://www.springer.com 